\title{
Substance P Modulates Colitis-Asscociated Fibrosis
}

\author{
Hon Wai Koon, ${ }^{*}$ David Shih, ${ }^{\dagger}$ \\ Iordanes Karagiannides, ${ }^{*}$ Dezheng Zhao, ${ }^{\ddagger}$ \\ Zafeer Fazelbhoy, ${ }^{*}$ Tressia Hing, ${ }^{*}$ Hua $\mathrm{Xu},{ }^{\neq}$ \\ Bao Lu, ${ }^{\S}$ Norma Gerard, ${ }^{\S}$ \\ and Charalabos Pothoulakis* \\ From the Inflammatory Bowel Disease Center," Division of \\ Digestive Diseases, David Geffen School of Medicine, University \\ of California at Los Angeles, Los Angeles, California; the \\ Inflammatory Bowel Disease Center and Immunobiology \\ Research Institute, ${ }^{\dagger}$ Cedars-Sinai Medical Center, Los Angeles, \\ California; the Center for Vascular Biology Research and \\ Division of Gastroenterology, ${ }^{\ddagger}$ Beth Israel Deaconess Medical \\ Center, Harvard Medical School, Boston, Massachusetts; the Ina \\ Sue Perlmutter Laboratory, ${ }^{\S}$ Pulmonary Division, the Department \\ of Pediatrics, Children's Hospital, Boston, Massachusetts; and the \\ Department of Medicine, " Harvard Medical School, Boston, \\ Massachusetts
}

Substance $P$ (SP) and the neurokinin-1 receptor (NK1R) are involved in the development of colitis and mucosal healing after colonic inflammation. We studied whether SP modulates colonic fibrosis by using a chronic model of trinitrobenzenesulfonic acid (TNBS)-induced colitis in wild-type (WT) and NK-1Rdeficient (NK-1R KD) mice. We found increased mRNA expression levels of collagen, vimentin, and the fibrogenic factors transforming growth factor $\beta 1$ and insulin-like growth factor 1 in the chronically inflamed colons of WT mice treated with repeated intracolonic TNBS administrations. Fibrosis in TNBStreated mice was also evident immunohistochemically by collagen deposition in the colon. Treatment of TNBS-exposed WT mice with the NK-1R antagonist CJ-12255 reduced colonic inflammation, colonic fibrosis, fibroblast accumulation, and expression levels of the fibrogenic factors. NK-1R knockout mice chronically exposed to TNBS had similar colonic inflammation compared with WT, but reduced colonic fibrosis, fibroblast accumulation, and expression levels of fibrogenic factors. Immunohistochemical staining also showed co-localization of NK-1R with fibroblasts in inflamed colons of mice and in colonic mucosa of patients with Crohn's disease. Exposure of human colonic CCD-18Co fibroblasts to SP (10 nmol/L) increased cell migration. SP stimulated collagen synthesis in CCD-18Co fibroblasts in the presence of transforming growth factor $\beta 1$ and insulin-like growth factor 1 , and this effect was reduced by Akt inhibition. Thus, SP, via NK-1R, promotes intestinal fibrogenesis after chronic colitis by stimulating fibrotic responses in fibroblasts. (Am J Pathol 2010, 177:2300-2309; DOI: 10.2353/ajpath.2010.100314)

Inflammatory bowel diseases, including Crohn's disease (CD) and ulcerative colitis, are chronic relapsing immune disorders that significantly impact health-related quality of life. ${ }^{1}$ Intestinal fibrosis is presented as excessive accumulation of fibrotic tissue in inflamed areas of the small intestine and colon of both ulcerative colitis and CD patients. ${ }^{2}$ As a result, patients with CD often develop transmural luminal narrowing and form strictures caused by excessive extracellular matrix deposition. ${ }^{3}$ Although the mechanisms involved in extracellular matrix deposition are complex and not well understood, it is well accepted that this process is driven by increased expression of collagen and other pro-fibrogenic factors such as transforming growth factor $\beta 1$ (TGF- $\beta 1$ ) and insulin-like growth factor 1 (IGF-1). ${ }^{4-6}$

Substance $P(\mathrm{SP})$ is an 11-amino acid neuropeptide belonging to the tachykinin family. ${ }^{7} \mathrm{SP}$ is expressed in the central nervous system, ${ }^{8}$ enteric nerves, ${ }^{9}$ sensory neurons, ${ }^{10}$ immune cell types, such as macrophages ${ }^{11,12}$ and T-lymphocytes, ${ }^{13}$ as well as in colonic epithelial cells. ${ }^{14-16}$ In the intestine SP binding to its high affinity

Supported in whole or in part by the National Institutes of Health grant DK47343 (C.P.), Al039759 (N.G.), a Research Fellowship Award by the Crohn's and Colitis Foundation of America, Inc (H.W.K. and I.K.), the Blinder Research Foundation for Crohn's Disease (H.W.K.), and the Eli and Edythe Broad Chair (C.P.). Pfizer, Inc. provided the NK-IR antagonist CJ-12255

Accepted for publication July 21, 2010

Address reprint requests to Charalabos Pothoulakis, M.D., Inflammatory Bowel Disease Center, Division of Digestive Diseases, David Geffen School of Medicine, University of California, Los Angeles, MRL Building, Room 1240, 675 Charles E. Young Dr. South, Los Angeles, CA 90095 E-mail: cpothoulakis@mednet.ucla.edu. 
receptor neurokinin-1 (NK-1) modulates several important intestinal functions, including colonic inflammation. ${ }^{17}$ Pharmacological antagonism of NK-1 receptor (NK-1R) or genetic deletion of this receptor results in reduced intestinal inflammation in the acute model of Clostridium difficile toxin A-induced enteritis or the acute phase of 2,4,6-trinitrobenzensulfonic acid (TNBS)-induced colitis. ${ }^{18-21}$ In contrast, NK-1R deficient (knockout [KO]) mice exposed to TNBS or sodium dextran sulfate, ${ }^{22}$ or mice treated with the NK-1R receptor antagonist CJ12255 during the healing phase of sodium dextran sulfate-induced colitis ${ }^{23}$ developed more severe colitis than control mice suggesting that NK-1R is also involved in mucosal healing during chronic colitis. However, there are no studies examining whether SP, or any other neuropeptide, is involved in colonic fibrosis.

Based on the ability of SP and NK-1R to modulate colonic inflammation and fibrosis through its interaction with fibroblasts, ${ }^{22,24}$ we hypothesized that SP/NK-1R interactions participate in colitis-associated fibrosis. To address our hypothesis, we used NK-1R KO and wild-type (WT) mice injected with the specific nonpeptide NK-1R antagonist CJ 12255 and the murine model of chronic TNBS-induced colitis associated with intestinal fibrosis. ${ }^{25}$ Our results indicate that WT mice treated with the NK-1R antagonist as well as NK-1R KO mice have reduced colonic fibrosis, fibroblast accumulation, and expression of fibrogenic factors in the colonic mucosa. NK-1R was co-localized with IGF-1 in colonic mucosa of patients with $\mathrm{CD}$ and TNBS-treated mice. Furthermore, in vitro experiments with human colonic CCD-18Co fibroblasts showed that SP stimulated fibroblast migration and, in the presence of TGF- $\beta 1$ and IGF-1, increased collagen synthesis in an Akt-dependent manner.

\section{Materials and Methods}

\section{Quantitative Real-Time RT-PCR}

Total RNA was isolated by Trizol Reagent (Invitrogen, Carlsbad, CA) and reverse-transcribed into cDNA by using a Superscript III reverse transcription kit (Invitrogen). Quantitative PCR reactions were run in an $A B I$ Prism 7700 Sequence Detection System as we previously described. ${ }^{26}$ The levels of mRNA were determined by using cataloged primers (Applied Biosystems, Foster City, CA) for human (COL1A2 Hs01028956_m1) and mouse (Col1a2 Mm01309565_m1; vimentin Mm00449208_ m1; Igf-1 Mm00439561-m1; and Tgf- $\beta 1$ Mm00441724_m1). Expression of these genes was normalized to expression of GAPDH/18S mRNA (human, 18S Hs99999901_s1; mouse, GAPDH Mm99999915_g1) and the results were expressed as relative fold differences.

\section{TNBS-Induced Colonic Fibrosis Mouse Model}

NK-1R KO mice were generated by targeted disruption of the NK-1R gene in embryonic stem cells as we previously described. ${ }^{27}$ Aged matched NK-1R KO and WT littermates (10- to 12-week-old) were maintained at the animal research facility of the University of California at Los Angeles. All animal studies were approved by the institutional animal care and use committee of the University of California, Los Angeles. Mice received standard pelleted chow and tap water ad libitum. Chronic TNBS colitis was induced as previously reported. ${ }^{25}$ Briefly, mice were injected intracolonically with a $50-\mu$ l of $30 \%$ ethanol containing a TNBS solution or $30 \%$ ethanol weekly for 5 weeks as illustrated in Figure 1A. Some groups were injected intraperitoneally with the specific nonpeptide NK-1R antagonist CJ-12255 (Pfizer, New York, NY; 5 $\mathrm{mg} / \mathrm{kg}$ in $100 \mu \mathrm{l}$ PBS, every 3 days during the last 4 weeks of the 8-week experimental period). Other groups were injected intraperitoneally with the same volume of PBS. Mice were then returned to their cages and sacrificed at week 8 by carbon dioxide euthanasia.

\section{Histological Scoring}

Colon tissues of mice were sectioned and stained with $\mathrm{H} \& \mathrm{E}$. Microphotographs at $200 \times$ magnification were recorded at multiple locations of colons and analyzed by two pathologists in a blinded manner who scored the specimens at a 0 to 12 scale for the following chronic colitis parameters: mucosal transformation, round cell infiltrates in the lamina propria mucosae, goblet cell death, submucosa fibrosis, and granuloma formation as previously established. ${ }^{28}$ Zero $=$ no inflammation; 1 to 4 = mild inflammation; 5 to $8=$ moderate inflammation; and 9 to $12=$ severe inflammation.

\section{Immunofluorescence Staining}

Human colonic tissue frozen sections were purchased from Cytomyx, Inc. (Rockville, MD). Colonic tissues were embedded in OCT solution, and frozen sections were made and fixed and permeabilized by using acetone. After incubation with blocking buffer, slides were incubated with a rabbit polyclonal rabbit anti-NK-1R antibody (sc-15323) or goat anti-vimentin antibody (sc-7557; Santa Cruz biotechnology, Santa Cruz, CA; all at 1:50 dilution) overnight at $4^{\circ} \mathrm{C}$. Samples were then washed with PBS and stained by Texas Red (red)-conjugated and fluorescein isothiocyanate (green)-conjugated secondary antibodies (1:200 dilution) for 1 hour. Slides were then rinsed and mounted with 4',6-diamidino-2-phenylindole (DAPI; blue emission signal to stain nuclei) mounting solution. Images were analyzed with a Zeiss (Thornwood, NY) AX10 microscope at $200 \times$ magnification.

\section{Immunohistochemistry for the Fibroblast Marker Vimentin}

Colon tissues were fixed in $4 \%$ paraformaldehyde and embedded in paraffin. After incubation with blocking buffer, sections were incubated with a goat polyclonal anti-vimentin antibody (sc-7557; Santa Cruz; 1:50 dilution) overnight at $4^{\circ} \mathrm{C}$. After washing, sections were incubated with donkey anti-goat IgG, and slides were stained 
A

TNBS intracolonic administration

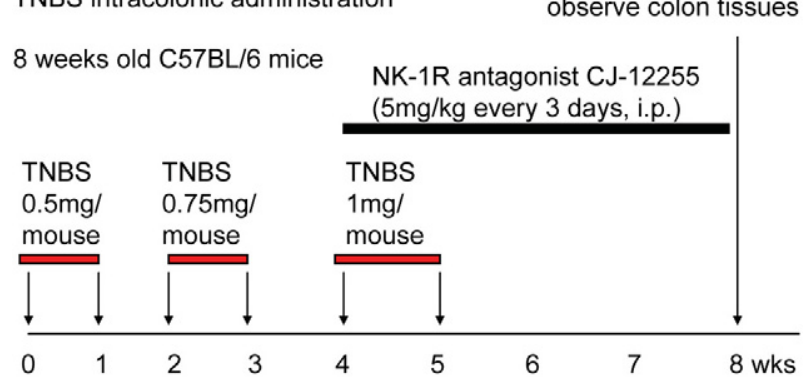

B

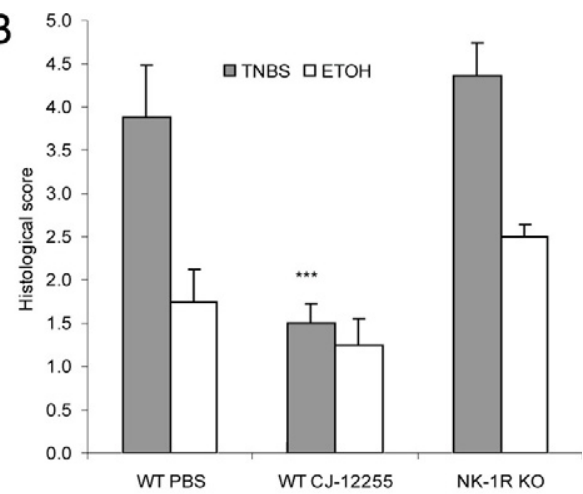

C

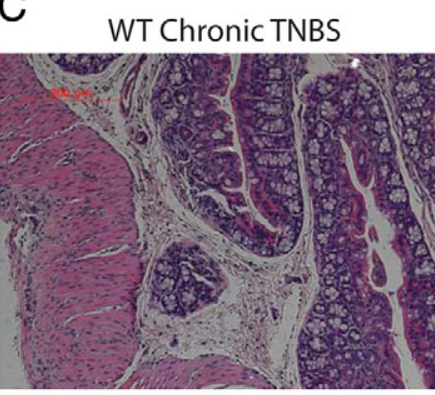

WT EtOH

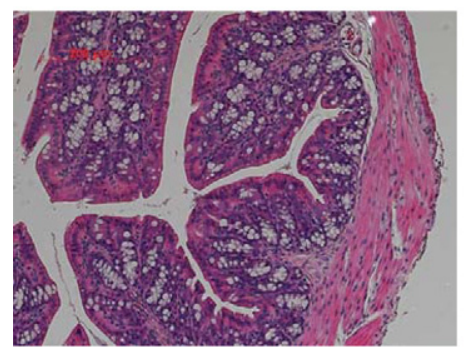

WT Chronic TNBS + CJ12255

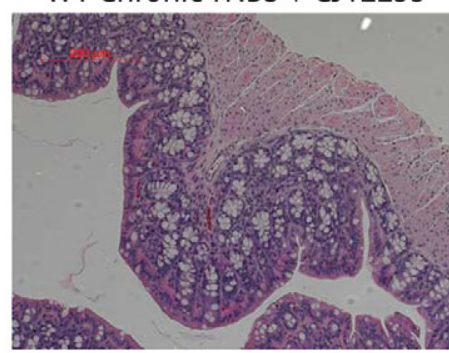

WT EtOH + CJ12255

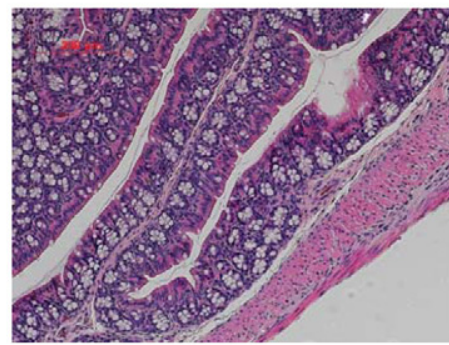

H\&E staining (200X)

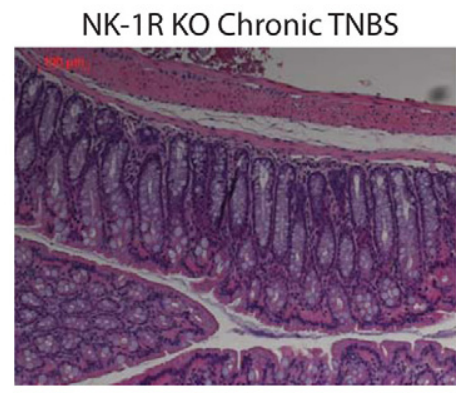

NK-1R KO EtOH

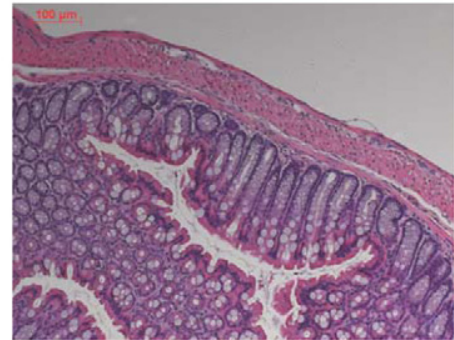

Figure 1. Chronic TNBS colitis model with colonic fibrosis. A: Schematic diagram of the method for the chronic TNBS colitis mouse model used to induce colonic fibrosis. Mice were injected with increasing doses of TNBS solution or ethanol vehicle intracolonically. Some groups received i.p. injections with the NK-1R antagonist CJ-12255. B: Histological score ( $n=4$ mice per group). Moderate histological damage in TNBS-exposed mice is reduced by CJ-12255 treatment. **** $P<$ 0.001 versus WT PBS TNBS colitis group. C: Representative microphotographs of H\&E-stained colon tissues (original magnification, $\times 200$ ). Substantial tissue damage and fibrotic tissue infiltration is evident after TNBS treatment in WT mice. Tissue morphology is improved in CJ-12255-treated TNBS-exposed mice, but not in TNBS-treated NK-1R KO mice.

with an avidin-biotin complex kit for color development (Santa Cruz; sc-2018). Images were analyzed with a Zeiss AX10 microscope at magnification of 200X.

\section{Masson Trichrome Staining}

Staining of collagen deposition of deparaffinized tissue sections was performed by a Masson Trichrome Staining kit (HT-10) and Bouin solution (HT-10132; Sigma, St. Louis, MO) following the manufacturer's instructions.

\section{Cell Culture}

Human CCD-18Co colonic fibroblasts were cultured in minimal essential medium Eagle's medium (ATCC, Manassas, VA) containing 10\% fetal bovine serum (Invitrogen) and 1\% penicillin/streptomycin (Invitrogen).

\section{IGF- 1 and TGF- $\beta 1$ Enzyme-Linked Immunosorbent Assays}

The levels of mouse IGF- 1 and TGF- $\beta 1$ levels were measured by the enzyme-linked immunosorbent assay kits (IGF-1 DY291 and TGF- $\beta 1$ DY791; R\&D Systems, Minneapolis, MN) according to the manufacturer's instructions.

\section{Cell Migration Assay}

Cell migration assays of CCD-18Co fibroblasts were performed by using a modified Boyden chamber approach (number ECM508; Millipore, Billerica, MA) as we previously described. ${ }^{26,29}$ Briefly, 0.1\% Trifluoroacetic acid (TFA), SP (number 05-23-0600; Calbiochem, Gibbstown, NJ), IGF-1 (number 407240; Calbiochem), or TGF- $\beta 1$ (number 616455; Calbiochem) was added into the lower 
chamber. CCD-18Co fibroblasts $\left(2.5 \times 10^{4}\right.$ cells $)$ were seeded in the upper chamber and incubated for 6 hours at $37^{\circ} \mathrm{C}$. Fibroblasts that had migrated through the membrane were stained according to the manufacturer's protocol, and migrated cells were determined by absorbance at $650 \mathrm{~nm}$.

\section{Cell Viability Assay}

CCD-18Co fibroblasts, seeded on 96-well plates $\left(10^{6}\right.$ cells/plate), were pretreated with $1 \mu \mathrm{mol} / \mathrm{L}$ CJ-12255 or vehicle for 30 minutes and then exposed to SP (10 $\mathrm{nmol} / \mathrm{L})$ or vehicle $(0.01 \%$ TFA). After 24 hours, $20 \mu \mathrm{l}$ of CellTiter AQeuous One solution (MTS tetrazolium compound, G3580; Promega, Madison, WI) was added, cells were incubated at $37^{\circ} \mathrm{C}$ for 1 hour, and absorbance at $490 \mathrm{~nm}$ (indicating cell viability) was measured on a 96-well plate reader as we described. ${ }^{30}$

\section{Western Blot Analyses}

Cells were lysed in $1 \times$ blue loading buffer (Cell Signaling, Danvers, MA). Equal amount of cell extracts were fractioned by $10 \%$ sodium dodecyl sulfate polyacrylamide gel electrophoresis, and proteins were transferred onto nitrocellulose membranes (400 mA for 2 hours at $4^{\circ} \mathrm{C}$; Bio-Rad, Hercules, CA). Membranes were blocked in 5\% nonfat milk in Tris-buffered saline Tween 20 (TBST; 50 $\mathrm{mmol} / \mathrm{L}$ Tris, $\mathrm{pH} 7.5,0.15 \mathrm{M} \mathrm{NaCl}$, and 0.05\% Tween 20), and then incubated with antibodies against phospho-Akt (ser473) number 4060, $\beta$-actin number 4967, $\beta$-tubulin number 2146 (Cell Signaling), NK-1R (sc-15323), or collagen 1A2 (sc-28655). Horseradish peroxidase-labeled antibodies were detected by chemiluminescence (Fisher Scientific, Pittsburgh, PA), and signals were captured by using the luminescent image analyzer LAS4000 (Fujifilm, Tokyo, Japan).

\section{Statistical Analyses}

Results were expressed as mean \pm SEM and analyzed by using the Prism professional statistics software program (Graphpad, San Diego, CA). Student's t-tests with Mann-Whitney post tests were used for intergroup comparisons.

\section{Results}

\section{Involvement of NK-1R in Colonic Fibrosis Associated with Chronic, TNBS-Induced Colitis}

\section{Histological Evaluation}

Colonic fibrosis is characterized by collagen deposition and overexpression of fibrogenic factors. ${ }^{2,31}$ To investigate the role of NK-1R in the development of fibrosis, we used a murine model of chronic colitis that develops colonic fibrosis ${ }^{25}$ in NK-1R KO mice as well as WT mice treated with the NK-1R receptor antagonist CJ-12255. Details of this model are included in Materials and Meth- ods and illustrated in Figure 1A. As shown in Figures 1C and $2 \mathrm{~A}$, compared with vehicle administration, WT mice treated with multiple TNBS cycles develop substantial fibrosis in the mucosa and submucosa. In contrast, CJ12255 treated, TNBS-exposed mice have less mucosal and submucosal fibrosis (Figure $1 \mathrm{C}$ and $2 \mathrm{~A}$ ) and overall better mucosal tissue integrity. Quantification of these histological differences shown in Figure 1B as "histological score" show mild to moderate levels of colitis (histological score 4 to 5) after TNBS administration and a $\sim 60 \%$ reduction of colitis after $\mathrm{CJ}-12255$ administration $(P<0.001)$. Notably, histology (Figure $1 \mathrm{C})$ and histological scores (Figure 1B) of ethanol (vehicle)-treated mice are low and not affected by CJ-12255 treatment. Moreover, NK-1R KO mice treated with multiple TNBS cycles develop similar histological tissue damage and histological score as TNBS-treated WT mice (Figure 1, B and C). Similar to WT TNBS-treated mice, colonic sections from NK-1R KO mice exposed to TNBS show signs of chronic inflammation including mucosal transformation with altered cryptal architecture, immune cell infiltration, and occasional formation of granulomas (Figure 1, B and C). In contrast, NK-1R KO mice have substantially less coIonic fibrosis than TNBS-exposed WT littermates (Figure $2 A)$. These results indicate that NK-1R is involved in colonic fibrosis in murine model of chronic colitis.

\section{Involvement of NK-1R in Collagen Deposition and Fibroblast Infiltration During Chronic TNBS-Induced Colitis}

Collagen is secreted by fibroblasts and fibroblast-like cells ${ }^{32}$ and can be identified immunohistochemically by the fibroblast marker vimentin. ${ }^{33}$ Masson Trichrome staining in the mucosa and submucosa of WT mice after chronic TNBS treatment showed increased collagen formation (Figure 2A). Compared with controls, we observed an increased number of vimentin-positive cells in the colonic mucosa and submucosa of WT TNBS-treated mice (Figures 2B and 3A, arrows) in areas with increased collagen deposition (Figure 2A), suggesting that colonic fibroblasts mediate increased collagen secretion. Treating TNBS-exposed wild-type or NK-1R KO mice with CJ-12255 reduced collagen deposition (Figure 2A) and fibroblast infiltration (Figure 2B). This is correlated with a reduction of vimentin-positive cells in TNBS-exposed coIonic tissues that are treated with CJ-12255 (Figure 3B). NK-1R KO mice exposed to TNBS also have reduced vimentin-positive cells compared with WT (Figure 3B). The number of fibroblasts among ethanol-treated groups is low and not affected by CJ-12255 treatment or NK-1R deficiency (Figure 3B).

Consistent with prior observations, ${ }^{25}$ WT mice treated with multiple TNBS cycles demonstrate high mRNA levels of collagen and vimentin, compared with ethanol control (Figure 3, C and D). NK-1R antagonism by CJ-12255 significantly lowers collagen and vimentin mRNA levels (Figure 3, C and D). Moreover, NK-1R KO mice have also lower collagen and vimentin mRNA after TNBS exposure 

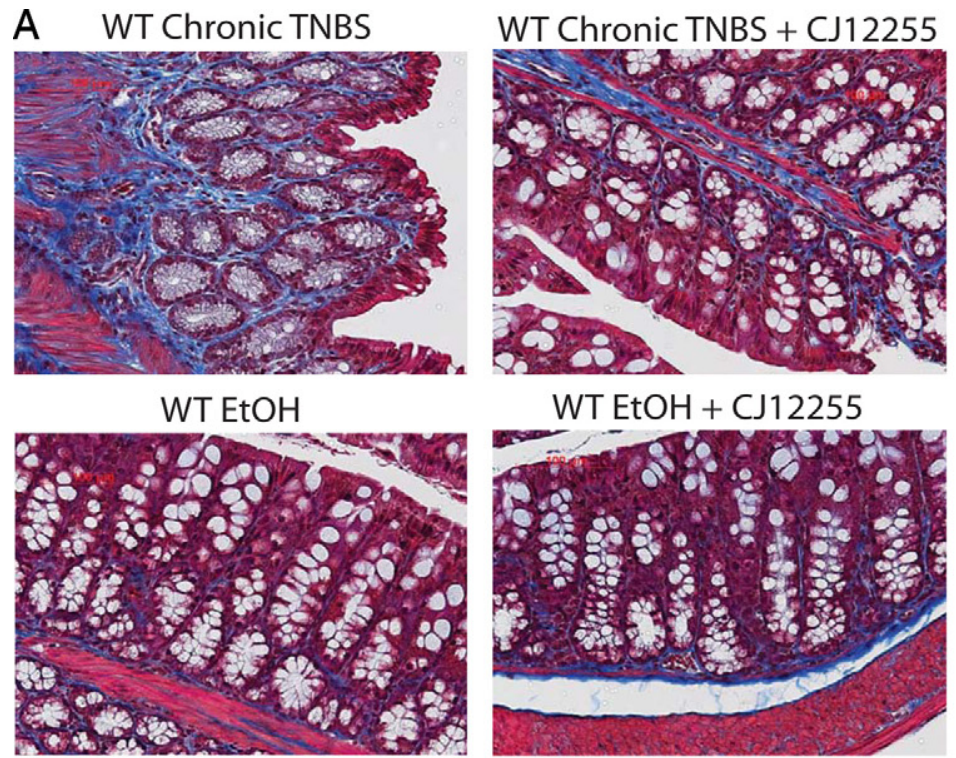

WT EtOH + CJ12255

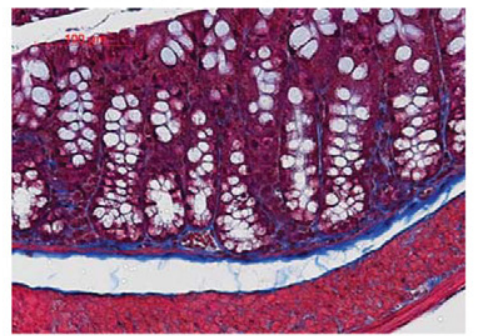

Masson Trichrome staining for collagen deposition

B WT Chronic TNBS

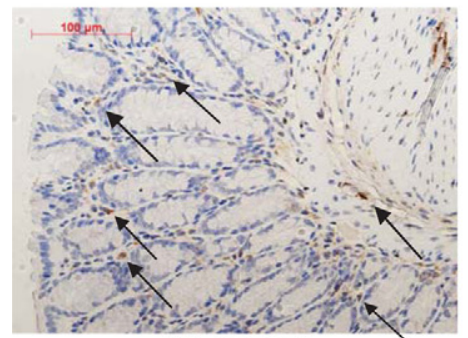

WT EtOH

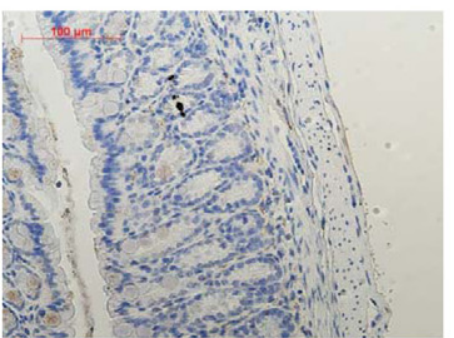

WT Chronic TNBS + CJ12255

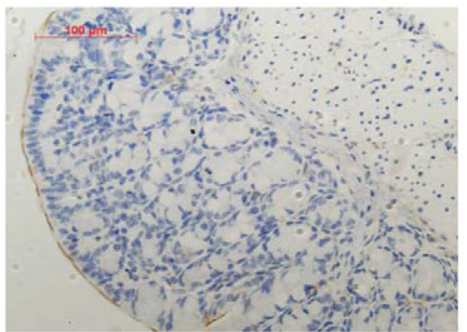

WT EtOH + CJ12255

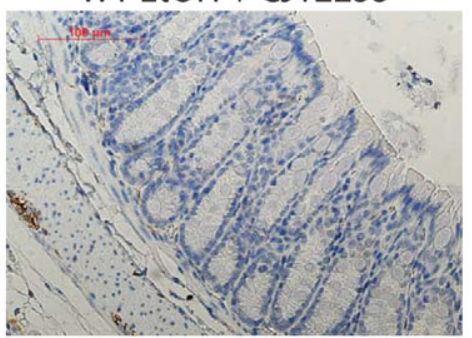

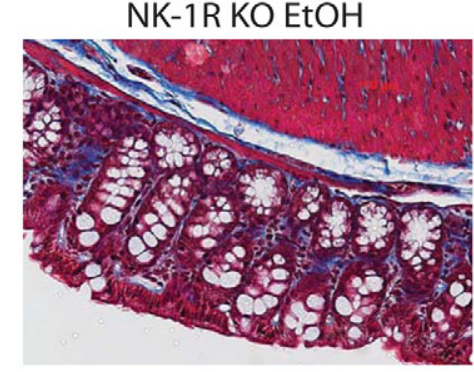

NK-1R KO Chronic TNBS

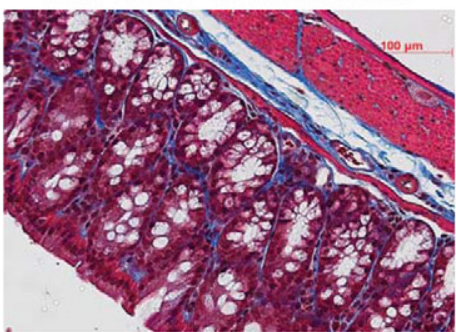

NK-1R KO EtOH

NK-1R KO Chronic TNBS

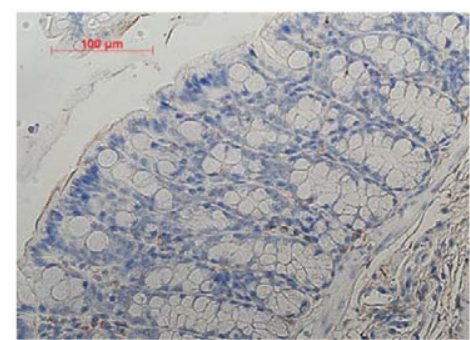

NK-1R KO EtOH

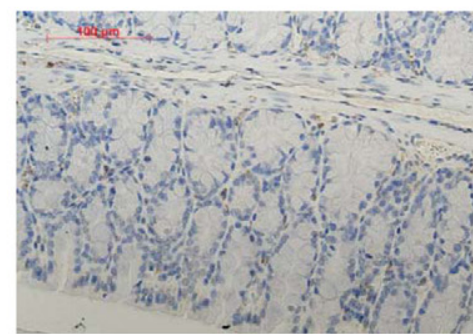

Vimentin (fibroblast marker) Immunohistochemistry

Figure 2. NK-1R mediates colonic fibrosis in vivo. Mice (PBS-treated WT, CJ-12255-treated WT, and NK-1R KO mice) were chronically treated with TNBS (CT) or $30 \%$ ethanol (EtOH) solution. A: Masson Trichrome staining of collagen deposition in tissue sections of mouse colon. Collagen is stained blue versus red background in TNBS-treated WT mice. There is reduced collagen in TNBS-exposed CJ-12255-treated mice and NK-1R KO mice. B: Immunohistochemical staining for the fibroblast marker vimentin. Fibroblasts are identified by specific staining (brown color) localized in the mucosa and submucosal in TNBS-exposed WT mice. Some fibroblasts are located by arrows in the figures. Tissue sections from TNBS-exposed CJ-12255-treated mice or NK-1R KO mice after TNBS administration have fewer vimentin-positive cells. Results are representative of four mice per group. Original magnification, $\times 200$.

(Figure 3, C and D), directly demonstrating an essential role for NK-1R in colitis-associated fibrogenesis.

\section{Role of NK-1R in Colonic Fibrogenic Factor Expression}

Increased colonic mRNA expression of fibrogenic factors TGF- $\beta 1$ and IGF- 1 in the colons of patients with CD has been reported previously. ${ }^{32,34}$ Consistent with these results, our experiments show increased TGF- $\beta 1$ and IGF- 1 colonic protein levels in inflamed colons of TNBS-treated mice (Figure 3, E and F). However, colonic levels of both TGF- $\beta 1$ and IGF- 1 are significantly lower in TNBS-exposed NK-1R KO mice and in WT mice treated with CJ-12255 (Figure 3, E and F).

\section{NK-1R Expression in Colonic Fibroblasts}

NK-1R is broadly expressed in the colons of patients with CD (Figure 4A), in line with previous reports. ${ }^{26,35}$ Double 
A

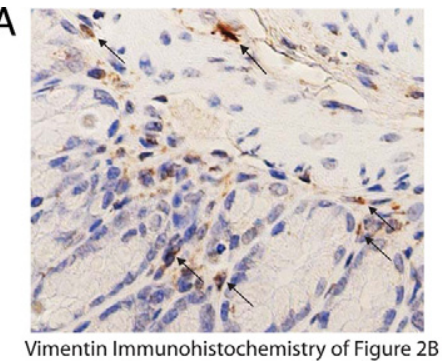

C

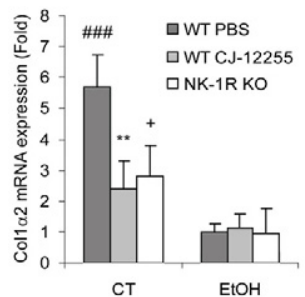

E

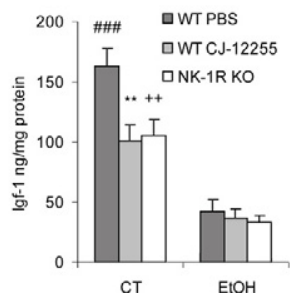

B

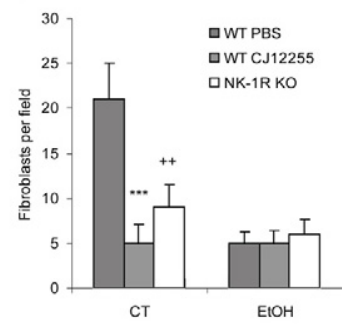

$\mathrm{D}$

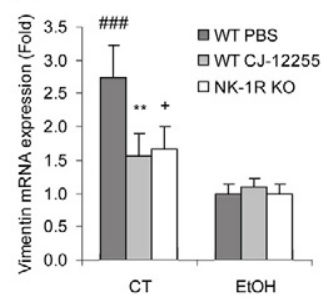

$\mathrm{F}$

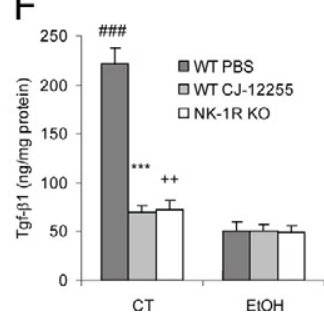

Figure 3. NK-1R mediates increased collagen, vimentin, IGF-1, and TGF- $\beta 1$ expression in inflamed colons. A: Magnified image of Figure $2 \mathrm{~B}$ showing location of vimentin-positive fibroblasts in the colons of WT chronic TNBS group clearly. Fibroblasts are identified by specific staining (brown color). Mice (PBS-treated WT, CJ-12255- treated WT, and NK-1R KO mice) were chronically treated with TNBS (CT) or 30\% ethanol (EtOH) solution. B: The number of fibroblasts in colon tissues in each microphotograph was counted as fibroblasts per field. A total of four pictures at random locations from each colonic tissue were counted and averaged. CJ-12255-treated mice and NK-1R KO mice have fewer fibroblasts in colons than TNBS-treated WT mice. ${ }^{* * * *} P<$ $0.001 ;{ }^{++} P<0.01$ versus WT PBS-treated chronic TNBS colitis (WT PBS) Results of real-time RT-PCR for detection of mouse Col1 $\alpha 2(\mathbf{C})$ and vimentin (D) mRNA levels in mouse colons. ${ }^{\# \#} P<0.001$ versus WT ethanol-treated (WT EtOH) group; ${ }^{* * *} P<0.01$ versus WT PBS-treated chronic TNBS colitis (WT PBS CT) group; ${ }^{+} P<0.05$ versus WT PBS CT group. Protein levels of mouse Igf-1 (E) and Tgf- $\beta 1(\mathbf{F})$ in mouse colons were measured by enzymelinked immunosorbent assay. ${ }^{\# \#} P<0.001$ versus WT ethanol-treated (WT EtOH) group; ${ }^{* * *} P<0.01,{ }^{* * * *} P<0.001$ versus WT PBS-treated chronic TNBS colitis (WT PBS CT) group; ${ }^{++} P<0.01$ versus WT PBS CT group. Colonic collagen and vimentin mRNA levels as well as IGF-1 and TGF- $\beta 1$ protein levels after TNBS exposure are reduced in CJ-12255-treated mice or NK-1R $\mathrm{KO}$ mice. Results are representative of four mice per group.

immunofluorescence staining show increased expression of vimentin (fibroblast marker) positive cells and colocalization of many of these cells with NK-1R expressing cells (Figure 4A). Similarly, we found strong widespread NK-1R staining in the colon of TNBS-treated mice, which is reduced after $\mathrm{CJ}-12255$ administration (Figure 4B). Several vimentin positive cells also express NK-1R in the colonic mucosa of TNBS-exposed mice (Figure 4B). Thus, colonic fibroblasts in human and mouse inflamed colon express NK-1R.

\section{Substance P Stimulates Colonic Fibroblast Migration}

To mediate colonic fibrosis, fibroblasts migrate to sites of inflammation where they deposit collagen. To determine

the role of $\mathrm{SP}$ in fibroblast migration, cultured human colonic CCD-18Co fibroblasts were seeded in the upper chamber of modified Boyden chambers. Addition of SP to the lower, but not upper, chamber induces significant fibroblast migration similar to that seen in response to TGF- $\beta 1$ and IGF- 1 serving as positive controls (Figure $5 A)$. We next tested whether SP induces proliferation in CCD-18Co cells. Addition of SP (10 nmol/L) alone does not increase cell proliferation, measured by the MTS assay (Figure 5B). SP also fail to stimulate CCD-18Co cell proliferation even in the presence of TGF- $\beta 1(1 \mathrm{ng} / \mathrm{ml})$ and IGF-1 (10 ng/ml; Figure 5B). Moreover, SP (10 nmol/ $L)$, either alone or in the presence of TGF- $\beta 1$ and IGF-1, does not affect TGF- $\beta 1$ or IGF- 1 mRNA expression (Figure $5, \mathrm{C}$ and $\mathrm{D}$ ).

\section{SP Induces Collagen Synthesis in Human Colonic Fibroblasts}

To determine whether SP affects fibrogenesis in vitro, we exposed human colonic CCD-18Co fibroblasts to SP in the presence or absence of TGF- $\beta 1$ and IGF-1. Incubation with SP alone for 48 hours does not induce collagen COL1A2 protein expression in CCD-18Co cells (data not shown). Exposure of fibroblasts to IGF-1 and TGF- $\beta 1$ does not increase expression of protein bands representing COL1A2 (Figure 6A). In the presence of TGF- $\beta 1$ and IGF-1, however, SP increases COL1A2 protein expression in a dose-dependent manner (Figure 6A). Similarly, $\mathrm{SP}$ in combination with TGF- $\beta 1$ and IGF-1, but not alone, induces COL1A2 mRNA expression (Figure 6B). In contrast, SP does not influence collagen COL1A2 mRNA expression when incubated with either TGF- $\beta 1$ alone or IGF-1 alone (Figure 6B). TGF- $\beta 1$ alone, serving as positive control, moderately increases collagen COL1A2 mRNA levels that is further augmented by IGF-1 coexposure in CCD-18Co fibroblasts, consistent with prior observations $^{6}$ (Figure 6B).

\section{SP Induces Collagen Synthesis in Human Colonic Fibroblasts via Akt}

IGF-1 induces Akt phosphorylation in lung fibroblasts, ${ }^{36}$ whereas SP activates Akt in colonic epithelial NCM460NK-1R cells. ${ }^{23}$ We examined whether SP induces collagen synthesis in fibroblasts by activating Akt. SP (10 $\mathrm{nmol} / \mathrm{L}$ ) alone does not stimulate Akt phosphorylation in colonic fibroblasts (Figure 6C). TGF- $\beta 1$ and IGF- 1 trigger weak Akt phosphorylation that was augmented by 10 $\mathrm{nmol} / \mathrm{L}$ of SP (Figure 6C). Furthermore, SP-induced collagen mRNA synthesis in the presence of TGF- $\beta 1$ and IGF-1 is also blocked by the Akt inhibitor $V$ (Figure 6D). These results indicate an important role for SP-augmented IGF-1/TGF- $\beta 1$ signaling pathways in collagen synthesis.

\section{Discussion}

Fibrostenosing disease in inflammatory bowel disease is a debilitating consequence of chronic colonic inflamma- 
A

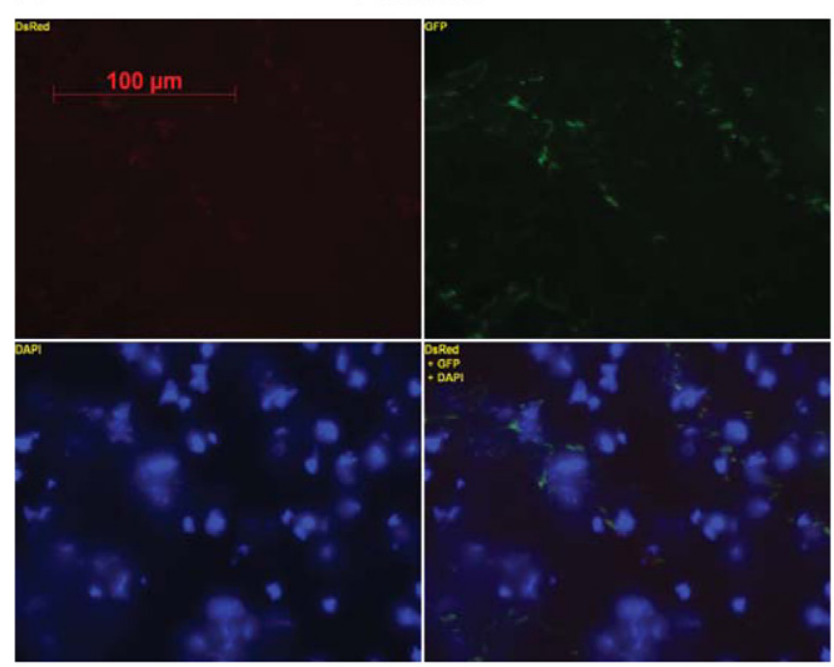

B

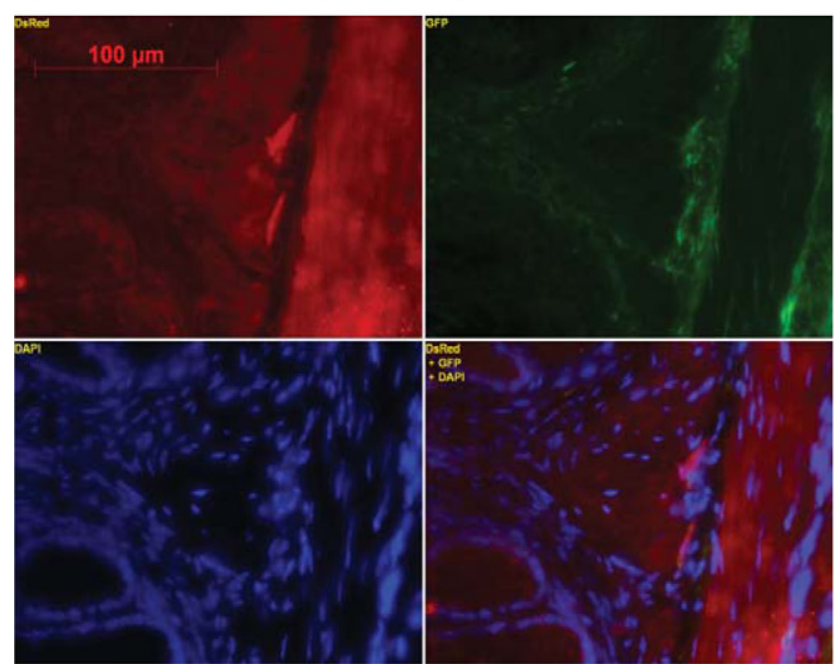

Crohn's Disease

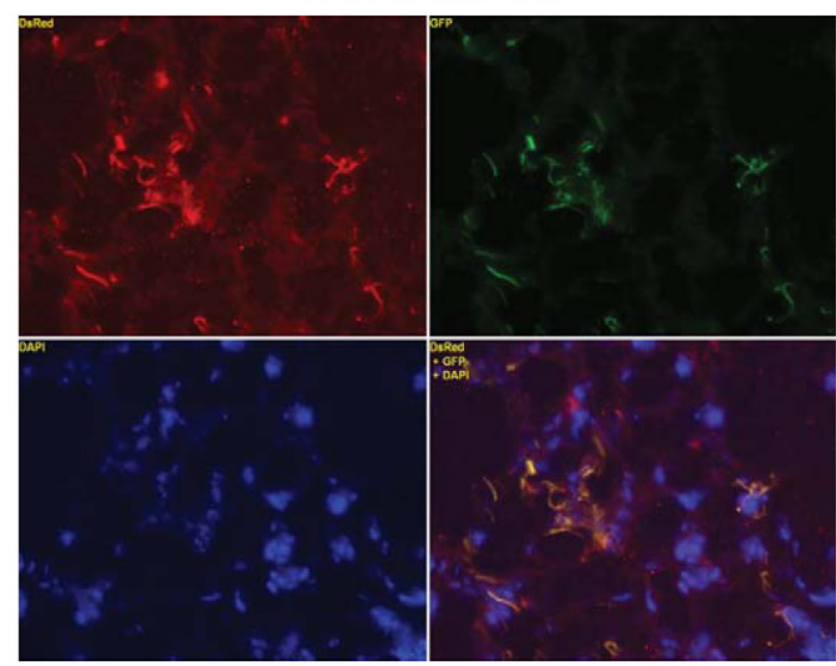

Chronic TNBS colitis + CJ-12255

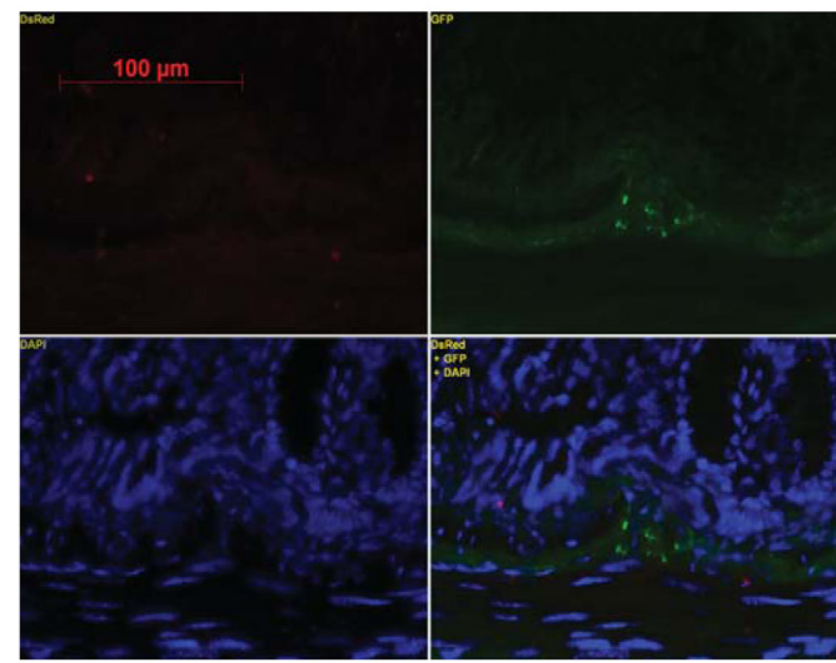

Figure 4. Colocalization of fibroblasts with NK-1R in the mucosa of chronic mouse colitis and CD colons. Double immunofluorescence staining of NK-1R (red) and fibroblast marker vimentin (green) counterstained by nuclear DAPI (blue) label in the colons of CD and normal patients (original magnification, $\times 400$; A) as well as colons from chronic TNBS treatment (CT) and CT with CJ-12255 administration (B; original magnification, $\times 400)$. Strong staining of NK-1R is found in the colon of CD and chronic TNBS (CT) groups. Merged images (yellow) indicate colocalization of vimentin and NK-1R. Results are representative of two separate experiments including samples from four patients with CD, four normal patients, four mice from the CT group, and four mice from the CT + CJ-12255 group.

tion. In this study, we examined the putative role of the neuropeptide SP and the NK-1R in the development of fibrosis during chronic colitis and examined the mechanisms of this response. Using a specific NK-1R antagonist and NK-1R deficient mice, we demonstrate that increased expression of collagen and the fibrogenic factors TGF- $\beta 1$ and IGF- 1 during chronic TNBS-induced colitis involves NK-1R signaling. SP can directly trigger migration of human colonic fibroblasts and increase their production of collagen in combination with TGF- $\beta 1$ and IGF-1, thereby illustrating a mechanism for SP-dependent fibrogenesis. These results uncover a novel role of $\mathrm{SP}$ in the modulation of fibrogenesis during colitis and implicate a neuropeptide-fibroblast paradigm in colitisrelated fibrogenesis in inflammatory bowel disease pathophysiology.
Inhibition of SP-NK-1R coupling by an NK-1R antagonist results in diminished intestinal inflammation. ${ }^{18,20,37}$ Not only NK-1R antagonism inhibits chronic colonic inflammation, but also reduces colonic fibrosis and expression of fibrogenic factors in vivo. The ability of NK-1R to signal both inflammatory and fibrotic responses is consistent with the view that intestinal fibrosis is closely associated with inflammation ${ }^{2}$ and mucosal healing after inflammation in the intestine. ${ }^{38}$ Our results demonstrate reduced TNBS-associated fibrosis in NK-1R KO mice and provide direct evidence for the importance of NK-1R in colitis-associated fibrosis. Despite reduced fibrosis in NK-1R KO mice, NK-1R deficiency did not alter the degree of chronic colonic inflammation in chronic TNBSinduced colitis. These results are consistent with previous studies in NK-1R KO mice using different models of co- 
A

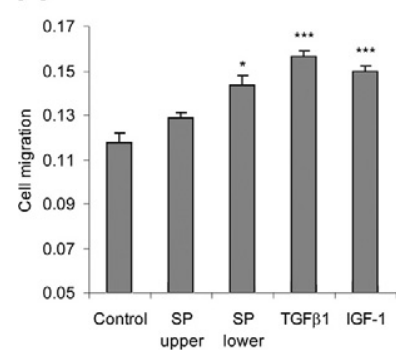

C

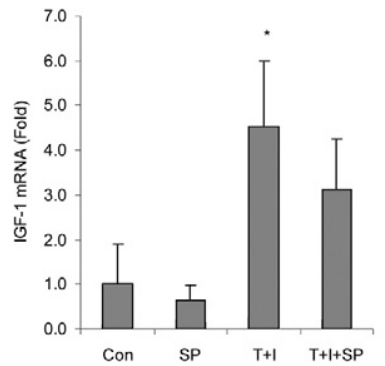

B

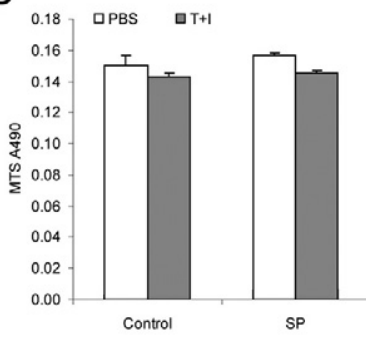

D

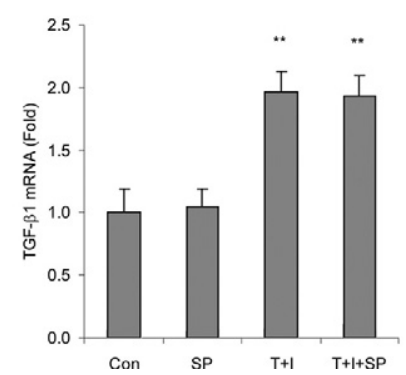

A
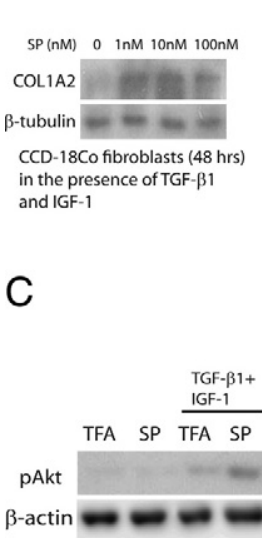

CCD-18Co fibroblasts $(30 \mathrm{~min})$
B

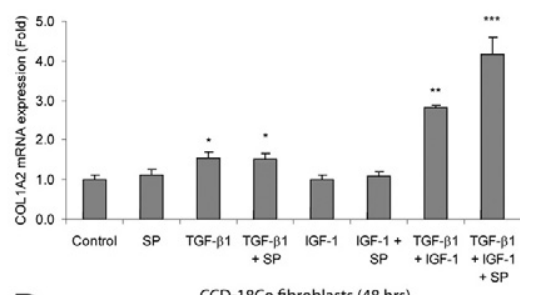

C CCD-18Co fibroblasts (48 hrs)

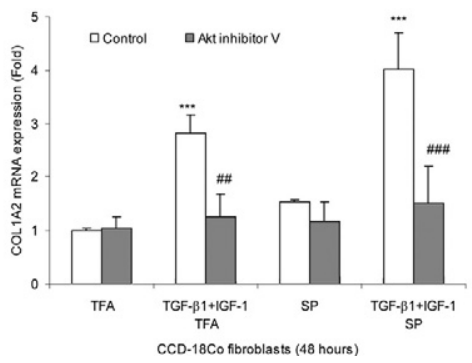

Figure 6. SP augments fibrogenesis in fibroblasts. A: CCD-18Co fibroblasts were treated with IGF-1 $(10 \mathrm{ng} / \mathrm{ml})$, TGF- $\beta 1(1 \mathrm{ng} / \mathrm{ml})$, and SP $(0$ to 100 $\mathrm{nmol} / \mathrm{L})$ at $37^{\circ} \mathrm{C}$ for 48 hours. Collagen COL1A2 in protein lysates was determined by Western blot analyses. B: CCD-18Co fibroblasts were treated with IGF-1 (10 ng/ml), TGF- $\beta 1(1 \mathrm{ng} / \mathrm{ml})$, and/or SP $(10 \mathrm{nmol} / \mathrm{L})$ at $37^{\circ} \mathrm{C}$ for 48 hours. Collagen COL1A2 mRNA levels were measured by real-time RTPCR. ${ }^{*} P<0.05,{ }^{* * *} P<0.01,{ }^{* * * * *} P<0.001$ versus control group. C: Fibroblasts were treated with IGF-1 (10 ng/ml), TGF- $\beta 1$ ( $1 \mathrm{ng} / \mathrm{ml})$, and/or SP $(10 \mathrm{nmol} / \mathrm{L})$ for 30 minutes, and phosphorylated Akt and $\beta$-actin in the protein lysates was determined by Western blot analyses. D: CCD-18Co cells were pretreated with dimethyl sulfoxide or Akt inhibitor $\mathrm{V}(5 \mu \mathrm{mol} / \mathrm{L})$ for 30 minutes, followed by IGF-1 (10 ng/ml), TGF- $\beta 1(1 \mathrm{ng} / \mathrm{ml})$, and/or SP $(10 \mathrm{nmol} / \mathrm{L})$ exposure at $37^{\circ} \mathrm{C}$ for 48 hours. Collagen COL1A2 mRNA expression was determined by real-time RT-PCR. Results are representative of three separate experiments. ${ }^{* * * *} P<0.001$ versus TFA-treated control group. ${ }^{\# \# P} P<0.01$

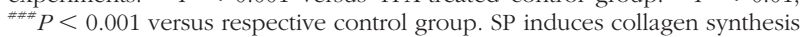
in the presence of IGF-1 and TGF- $\beta 1$ via an Akt-dependent pathway.

NK-1R stimulates proliferation of mouse colonic fibroblasts. ${ }^{22}$ On the other hand, SP alone also failed to stimulate proliferation in human skin fibroblasts. ${ }^{45}$ Different fibroblast cell phenotypes may explain these discrepant results.

We found that SP, in the presence of TGF- $\beta 1$ and IGF-1, stimulates collagen synthesis in colonic fibroblasts in vitro (Figure 6), supporting an NK-1R-dependent regulation of collagen synthesis during chronic TNBS colitis (Figures 2 and 3). However, NK-1R-regulated TGF- $\beta 1$ and IGF-1 expression in vivo (Figure 3, $\mathrm{E}$ and $\mathrm{F}$ ) has not been observed in CCD-18Co fibroblast culture (Figure 5, C and D) and NCM460 colonic epithelial cells overexpressing NK-1R (data not shown) in vitro. It is possible that SP-NK-1R interactions may influence fibrogenic growth factors regulation in the presence of other proinflammatory mediators present in animal models of colitis as suggested by prior studies. ${ }^{46,47}$ Different cell types may respond differently to SP since, in contrast to our results indicating lack of a direct SP collagen response in the absence of TGF- $\beta 1$ and IGF-1, lung fibroblasts have reduced collagen expression after SP exposure. ${ }^{48}$ Nevertheless, our evidence indicating the presence of NK-1R in colonic fibroblasts in inflamed colons (Figure 4) and SPresponsive signaling in CCD-18Co fibroblasts (Figure 6) strongly suggest an important role of SP-NK-1R interactions in colonic fibrogenesis. 
Our results show that increased collagen synthesis by $\mathrm{SP}$ in the presence of TGF- $\beta 1$ and IGF-1 involves the Akt pathway. IGF-1 is known to induce Akt phosphorylation via an IGF-1 receptor-dependent mechanism in mouse embryo fibroblasts. ${ }^{49}$ IGF-1 also induces Akt phosphorylation and protects lung myofibroblasts from apoptosis. ${ }^{50}$ Consistently, we previously reported that SP-mediated anti-apoptotic effects in colonic epithelial cells involve a phosphatidylinositol 3-kinase- and Akt-dependent pathway. ${ }^{23}$

In conclusion, these data demonstrate that inhibition of NK-1R can decrease colitis-associated intestinal fibrosis by modulating fibroblast migration and collagen synthesis. The results presented in this study add NK-1R as an important contributor to the pathophysiology of fibrosis complicating intestinal inflammation and point to a new possible target for prevention of fibrosis during colitis.

\section{Acknowledgment}

We thank Pfizer, Inc. for generously providing the NK-1R antagonist CJ-12255.

\section{References}

1. Shih DQ, Targan SR: Insights into IBD Pathogenesis. Curr Gastroenterol Rep 2009, 11:473-480

2. Rieder F, Fiocchi C: Intestinal fibrosis in IBD: a dynamic, multifactorial process. Nat Rev Gastroenterol Hepatol 2009, 6:228-235

3. Fiocchi C: Inflammatory bowel disease: etiology and pathogenesis. Gastroenterology 1998, 115:182-205

4. Di Sabatino A, Jackson CL, Pickard KM, Buckley M, Rovedatti L, Leakey NA, Picariello L, Cazzola P, Monteleone G, Tonelli F, Corazza GR, MacDonald TT, Pender SL: Transforming growth factor beta signalling and matrix metalloproteinases in the mucosa overlying Crohn's disease strictures. Gut 2009, 58:777-789

5. Fichtner-Feigl S, Young CA, Kitani A, Geissler EK, Schlitt HJ, Strober W: IL-13 signaling via IL-13R alpha2 induces major downstream fibrogenic factors mediating fibrosis in chronic TNBS colitis. Gastroenterology 2008, 135:2003-2013

6. Simmons JG, Pucilowska JB, Keku TO, Lund PK: IGF-I and TGF-beta1 have distinct effects on phenotype and proliferation of intestinal fibroblasts. Am J Physiol Gastrointest Liver Physiol 2002, 283:G809-G818

7. Chang MM, Leeman SE: Isolation of a sialogogic peptide from bovine hypothalamic tissue and its characterization as substance P. J Biol Chem 1970, 245:4784-4790

8. Mantyh PW: Neurobiology of substance P and the NK1 receptor. J Clin Psychiatry 2002, 63 Suppl 11:6-10

9. Costa M, Furness JB, Franco R, Llewellyn-Smith I, Murphy R, Beardsley AM: Substance $P$ in nerve tissue in the gut. Ciba Found Symp 1982, 129-144

10. Maggi CA: Capsaicin-sensitive nerves in the gastrointestinal tract. Arch Int Pharmacodyn Ther 1990, 303:157-166

11. Bost KL: Quantification of macrophage-derived substance P receptor mRNA using competitive polymerase chain reaction. Adv Exp Med Biol 1995, 373:219-223

12. Castagliuolo I, Keates AC, Qiu B, Kelly CP, Nikulasson S, Leeman SE, Pothoulakis $\mathrm{C}$ : Increased substance $\mathrm{P}$ responses in dorsal root ganglia and intestinal macrophages during Clostridium difficile toxin $\mathrm{A}$ enteritis in rats. Proc Natl Acad Sci USA 1997, 94:4788-4793

13. Blum A, Setiawan T, Hang L, Stoyanoff K, Weinstock JV: Interleukin-12 (IL-12) and IL-23 induction of substance p synthesis in murine $\mathrm{T}$ cells and macrophages is subject to $\mathrm{IL}-10$ and transforming growth factor beta regulation. Infect Immun 2008, 76:3651-3656

14. Koon HW, Zhao D, Zhan Y, Simeonidis S, Moyer MP, Pothoulakis C: Substance P-stimulated interleukin-8 expression in human colonic epithelial cells involves protein kinase Cdelta activation. J Pharmacol Exp Ther 2005, 314:1393-1400

15. Koon HW, Zhao D, Zhan Y, Rhee SH, Moyer MP, Pothoulakis C: Substance P stimulates cyclooxygenase-2 and prostaglandin E2 expression through JAK-STAT activation in human colonic epithelial cells. J Immunol 2006, 176:5050-5059

16. Simeonidis S, Castagliuolo I, Pan A, Liu J, Wang CC, Mykoniatis A, Pasha A, Valenick L, Sougioultzis S, Zhao D, Pothoulakis C: Regulation of the NK-1 receptor gene expression in human macrophage cells via an NF-kappa B site on its promoter. Proc Natl Acad Sci USA 2003, 100:2957-2962

17. Koon HW, Pothoulakis C: Immunomodulatory properties of substance P: the gastrointestinal system as a model. Ann NY Acad Sci 2006, 1088:23-40

18. Pothoulakis C, Castagliuolo I, LaMont JT, Jaffer A, O'Keane JC, Snider RM, Leeman SE: CP-96,345, a substance P antagonist, inhibits rat intestinal responses to Clostridium difficile toxin $A$ but not cholera toxin. Proc Natl Acad Sci USA 1994, 91:947-951

19. Mantyh CR, Pappas TN, Lapp JA, Washington MK, Neville LM, Ghilardi JR, Rogers SD, Mantyh PW, Vigna SR: Substance P activation of enteric neurons in response to intraluminal Clostridium difficile toxin A in the rat ileum. Gastroenterology 1996, 111:1272-1280

20. Stucchi AF, Shofer S, Leeman S, Materne O, Beer E, McClung J, Shebani K, Moore F, O'Brien M, Becker JM: NK-1 antagonist reduces colonic inflammation and oxidative stress in dextran sulfate-induced colitis in rats. Am J Physiol Gastrointest Liver Physiol 2000, 279:G1298-G1306

21. Castagliuolo I, Riegler M, Pasha A, Nikulasson S, Lu B, Gerard C Gerard NP, Pothoulakis C: Neurokinin-1 (NK-1) receptor is required in Clostridium difficile-induced enteritis. J Clin Invest 1998, 101:15471550

22. Castagliuolo I, Morteau O, Keates AC, Valenick L, Wang CC, Zacks J, Lu B, Gerard NP, Pothoulakis C: Protective effects of neurokinin-1 receptor during colitis in mice: role of the epidermal growth factor receptor. Br J Pharmacol 2002, 136:271-279

23. Koon HW, Zhao D, Zhan Y, Moyer MP, Pothoulakis C: Substance $P$ mediates antiapoptotic responses in human colonocytes by Akt activation. Proc Natl Acad Sci USA 2007, 104:2013-2018

24. Pucilowska JB, Williams KL, Lund PK: Fibrogenesis. IV. Fibrosis and inflammatory bowel disease: cellular mediators and animal models. Am J Physiol Gastrointest Liver Physiol 2000, 279:G653-G659

25. Lawrance IC, Wu F, Leite AZ, Willis J, West GA, Fiocchi C, Chakravarti $\mathrm{S}$ : A murine model of chronic inflammation-induced intestinal fibrosis down-regulated by antisense NF-kappa B. Gastroenterology 2003, 125:1750-1761

26. Koon HW, Zhao D, Xu H, Bowe C, Moss A, Moyer MP, Pothoulakis C: Substance P-mediated expression of the pro-angiogenic factor CCN1 modulates the course of colitis. Am J Pathol 2008, 173:400-410

27. Bozic CR, Lu B, Hopken UE, Gerard C, Gerard NP: Neurogenic amplification of immune complex inflammation. Science 1996, 273:1722-1725

28. Kruschewski M, Foitzik T, Perez-Canto A, Hubotter A, Buhr HJ Changes of colonic mucosal microcirculation and histology in two colitis models: an experimental study using intravital microscopy and a new histological scoring system. Dig Dis Sci 2001, 46:2336-2343

29. Koon HW, Kim YS, Xu H, Kumar A, Zhao D, Karagiannides I, Dobner PR, Pothoulakis C: Neurotensin induces IL-6 secretion in mouse preadipocytes and adipose tissues during 2,4,6,-trinitrobenzensulphonic acid-induced colitis. Proc Natl Acad Sci USA 2009, 106:8766-8771

30. Koon HW, Zhao D, Na X, Moyer MP, Pothoulakis C: Metalloproteinases and transforming growth factor-alpha mediate substance $\mathrm{P}$ induced mitogen-activated protein kinase activation and proliferation in human colonocytes. J Biol Chem 2004, 279:45519-45527

31. Burke JP, Mulsow JJ, O'Keane C, Docherty NG, Watson RW, O'Connell PR: Fibrogenesis in Crohn's disease. Am J Gastroenterol 2007, 102:439-448

32. Lawrance IC, Maxwell L, Doe W: Inflammation location, but not type, determines the increase in TGF-beta1 and IGF-1 expression and collagen deposition in IBD intestine. Inflamm Bowel Dis 2001, 7:16-26

33. Motomura Y, Khan WI, El-Sharkawy RT, Verma-Gandhu M, Verdu EF, Gauldie J, Collins SM: Induction of a fibrogenic response in mouse 
colon by overexpression of monocyte chemoattractant protein 1. Gut 2006, 55:662-670

34. Zimmermann EM, Li L, Hou YT, Mohapatra NK, Pucilowska JB: Insulin-like growth factor I and insulin-like growth factor binding protein 5 in Crohn's disease. Am J Physiol Gastrointest Liver Physiol 2001, 280:G1022-G1029

35. Goode T, O'Connell J, Anton P, Wong H, Reeve J, O'Sullivan GC, Collins JK, Shanahan F: Neurokinin-1 receptor expression in inflammatory bowel disease: molecular quantitation and localisation. Gut 2000, 47:387-396

36. Choi JE, Lee SS, Sunde DA, Huizar I, Haugk KL, Thannickal VJ, Vittal R, Plymate SR, Schnapp LM: Insulin-like growth factor-I receptor blockade improves outcome in mouse model of lung injury. Am $J$ Respir Crit Care Med 2009, 179:212-219

37. Stucchi AF, Shebani KO, Leeman SE, Wang CC, Reed KL, Fruin AB, Gower AC, McClung JP, Andry CD, O'Brien MJ, Pothoulakis C, Becker JM: A neurokinin 1 receptor antagonist reduces an ongoing ileal pouch inflammation and the response to a subsequent inflammatory stimulus. Am J Physiol Gastrointest Liver Physiol 2003, 285:G1259-G1267

38. Rieder F, Brenmoehl J, Leeb S, Scholmerich J, Rogler G: Wound healing and fibrosis in intestinal disease. Gut 2007, 56:130-139

39. Castagliuolo I, Valenick L, Liu J, Pothoulakis C: Epidermal growth factor receptor transactivation mediates substance P-induced mitogenic responses in U-373 MG cells. J Biol Chem 2000, 275: 26545-26550

40. Nakamura M, Nishida T, Ofuji K, Reid TW, Mannis MJ, Murphy CJ: Synergistic effect of substance $\mathrm{P}$ with epidermal growth factor on epithelial migration in rabbit cornea. Exp Eye Res 1997, 65:321-329

41. Marion-Audibert AM, Nejjari M, Pourreyron C, Anderson W, Gouysse G, Jacquier MF, Dumortier J, Scoazec JY: Effects of endocrine pep- tides on proliferation, migration and differentiation of human endothelial cells. Gastroenterol Clin Biol 2000, 24:644-648

42. Tominaga K, Honda K, Akahoshi A, Makino Y, Kawarabayashi T, Takano Y, Kamiya H: Substance P causes adhesion of neutrophils to endothelial cells via protein kinase C. Biol Pharm Bull 1999, 22:1242-1245

43. Parenti A, Amerini S, Ledda F, Maggi CA, Ziche M: The tachykinin NK1 receptor mediates the migration-promoting effect of substance $P$ on human skin fibroblasts in culture. Naunyn Schmiedebergs Arch Pharmacol 1996, 353:475-481

44. Seo M, Lee WH, Suk K: Identification of novel cell migration-promoting genes by a functional genetic screen. FASEB J 2010, 24:464-478

45. Kahler CM, Herold M, Wiedermann CJ: Substance P: a competence factor for human fibroblast proliferation that induces the release of growth-regulatory arachidonic acid metabolites. J Cell Physiol 1993, 156:579-587

46. Katayama I, Nishioka K: Substance P augments fibrogenic cytokineinduced fibroblast proliferation: possible involvement of neuropeptide in tissue fibrosis. J Dermatol Sci 1997, 15:201-206

47. Reed KL, Stucchi AF, Leeman SE, Becker JM: Inhibitory effects of a neurokinin-1 receptor antagonist on postoperative peritoneal adhesion formation. Ann NY Acad Sci 2008, 1144:116-126

48. Ramos C, Montano M, Cisneros J, Sommer B, Delgado J, GonzalezAvila G: Substance $P$ up-regulates matrix metalloproteinase-1 and down-regulates collagen in human lung fibroblast. Exp Lung Res 2007, 33:151-167

49. Povsic TJ, Kohout TA, Lefkowitz RJ: Beta-arrestin1 mediates insulinlike growth factor 1 (IGF-1) activation of phosphatidylinositol 3-kinase (PI3K) and anti-apoptosis. J Biol Chem 2003, 278:51334-51339

50. Wynes MW, Frankel SK, Riches DW: IL-4-induced macrophage-derived IGF-I protects myofibroblasts from apoptosis following growth factor withdrawal. J Leukoc Biol 2004, 76:1019-1027 